\title{
DESCRIPTIVE EPIDEMIOLOGY OF A GASTROENTERITIS OUT BREAK IN SUNSARI DISTRICT, NEPAL
}

\author{
Sharma A K', Jha $\mathbf{N}^{1}$, Ramachandran $\mathbf{V} \mathbf{G}^{1}$ \\ Shariff $\mathbf{M}^{1}$, Deb $\mathbf{M}^{1}$, Kannan A $\mathbf{T}^{1}$, Paudel I $\mathrm{S}^{1}$, Yadav B $\mathrm{K}^{1}$
}

\section{ABSTRACT}

Gastroenteritis (GE) outbreak and cholera epidemics continue to be the major public health problems in Nepal. Every year during and immediately after the rainy season, outbreak of GE occurs. In the post monsoon period of 1998 also, outbreak of GE occurred in a wide area covering several districts in terai region. In this paper, several epidemiological aspects of the current outbreak are being reported. This study was carried out in sunsari district. The cases of GE brought to district hospital were interviewed by a trained health worker to collect information about the episode and possible source of infection. Stool samples were collected from the patients and tested for organisms. On the spot visits were made to collect more information. Water samples were also collected and tested in Microbiology laboratory, B. P. Koirala Institute of Health Sciences (BPKIHS). There were 947 cases of GE. Among them 55.2\% were females and $44.8 \%$ males patients. The age ranged from 7 months to 86 years with mean age of 22.8 years. There were 8 deaths. Maximum number of cases occurred in the first fornight of October 1998. Due to prior administration of antibiotics, no growth was seen in 27 samples. However, 4 samples showed growth of Shigella. Vibrio cholera was isolated from 2 cases. Eight out of thirteen water samples were found to be unsatisfactory for drinking. The contamination of drinking water source and unhygienic habits were the main causes for such outbreaks. Therefore, it is pertinent that GE outbreaks may be prevented by simply promoting sanitation and hygienic practices related to handling of drinking water and defecation.

1. B. P. Koirala Institute of Health Sciences, Dharan, Nepal.

Address for correspondence : Dr. Nilambar Jha, Assoc. Professor Dept. of Community Medicine

B. P. Koirala Institute of Health Sciences

Dharan, Nepal. 


\section{INTRODUCTION}

Gastroenteritis out break and cholera epidemics continue to be the major public health problems in the terai region of Nepal. Every year during and immediately after the rainy season, out break of Gastroenteritis (GE) occurs. Studies carried out in the past have shown $\mathrm{V}$ Cholera as the principle organism, 92,000 cases and 1,800 deaths were reported in 1991 gastroenteritis epidemic throughout Nepal. ${ }^{1}$ Neither have this been followed up with intervention programmes to reduce such events or control the confined to the Kathmandu valley only. ${ }^{2-4}$ Literature is lacking on the epidemiology of such out breaks in the thickly populated eastern tropical lowland of Nepal (Terai). In the post mansoon period of 1998 also, outbreak of gastroenteritis occurred in a wide area covering several districts in terai region. In this paper, several epidemiological aspects of the current outbreak are being reported.

\section{OBJECTIVES}

To study the distribution and determinants of Gastroenteritis outbreak in the terai region of eastern Nepal.

\section{METHODOLOGY}

This study was carried out in the Sunsari district. The cases of GE brought to the Sunsari District Hospital, Ineruwa formed the main sample. A questionnaire was administered to the patients by a trained health worker to collect information about the episode of the GE and possible source of infection among them. Stool samples were collected from patients admitted in the district hospital. Later on, visits were made to the Village Development Committee (VDC) from where large number of cases were being reported. In each such (VDC) stool samples were collected from at least two persons suffering from diarrhoea during the visit of the investigating team. Water samples were also collected from the Hand-pumps, which were used by the patients suffering from diarrhoea and their families.

\section{RESULTS}

In all 947 Cases were line listed in the District Hospital emergency register between 14 ${ }^{\text {th }}$ July 1998 and $14^{\text {th }}$ November 1998. There were 523 female (55.2\%) and 424 (44.8\%) male patients. The age ranged between 7 months and 86 years. However the mean age was 22.81 years $17.2 \%$ of the cases were under five children and another $13.7 \%$ in the 5 to 15 years age group (Table 1). However in each

Table I. Age sex distribution of the cases

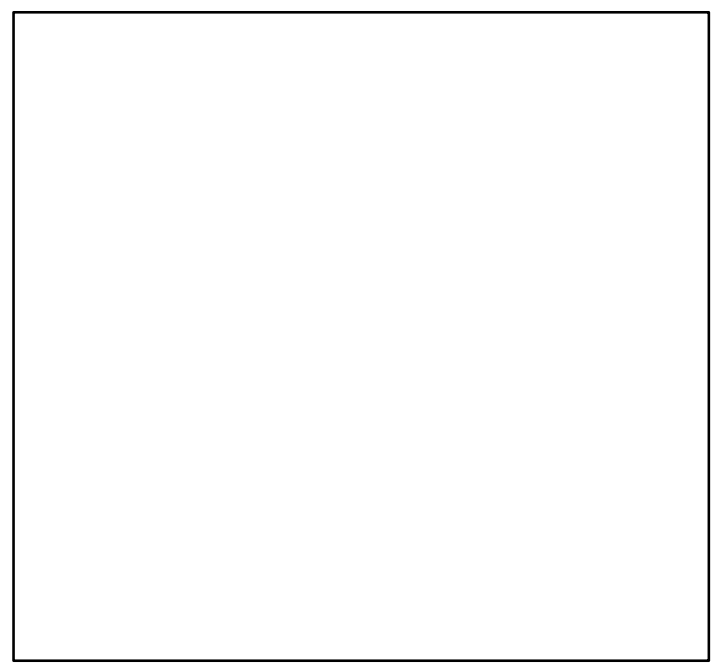

* Figures in parentheses indicate percentage age group the proportion of female cases was significantly more than that of the male cases except in children below 9 years of age. The geographical distribution of the cases is given in Fig.1. The maximum number of cases were from Ineruwa municipal area (22.6\%) followed by Dumraha VDC from which $12.0 \%$ cases had come. Sunsari district has 75 VDCs and cases were not reported from only 3 VDCs. Only one death occurred among the patients admitted in the 


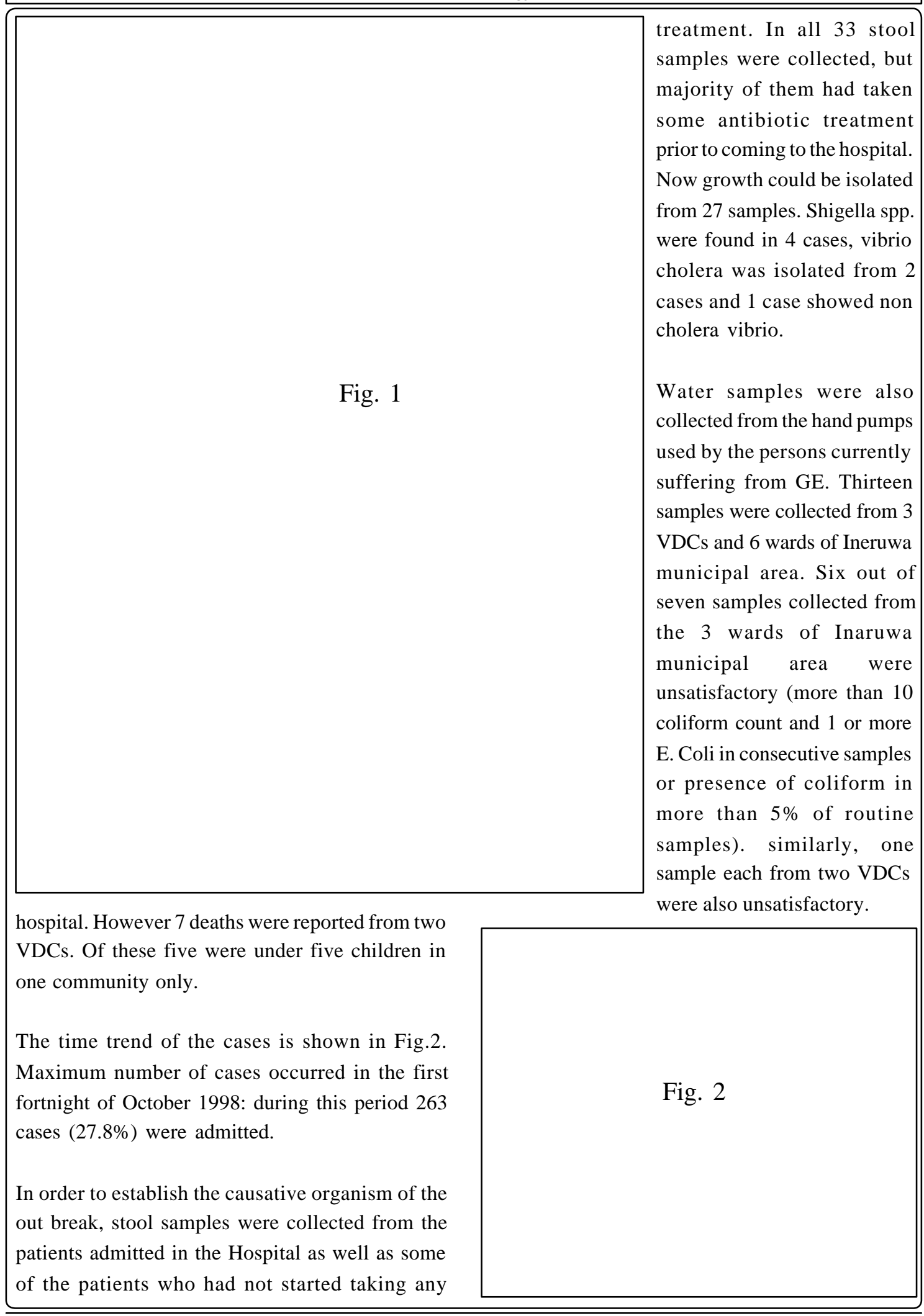

JNMA, July - September, 2002, 41 


\section{DISCUSSION}

The health care delivery and management of the epidemics is primarily the job of district health office. The BPKIHS was asked to help in investigation and control of the epidemic at a very late stage when the number of cases reporting to the district hospital had gone up considerably. i.e. around last week of September. Hence valuable time was lost in the process. However, the case distribution suggests that the epidemic had peaked in the first fortnight of October 1998 only.

The geographical distribution of cases was wide and scattered. Fig.1 reveals that cases were reported from almost all the VDCs of the district barring three which were at the foot bills and the District hospital was far from those VDCs. Cases from these three VDCs might have been treated by private practitioners or the nearby teaching hospital associated with BPKIHS. But cases were brought to the Ineruwa hospital from other adjacent districts viz. Siraha, Morang and Saptari also. Out break was also reported during the same period from other districts in the terai region.

The maximum number of cases occurring in the younger age group (under 14 years) points at the fact that lack of hygienic practices must have been one of the key factors. Informal interview with the village leaders and residents revealed that the practice of washing hands after defection was unsatisfactory. Majority of the people use mud for washing hands. Use of soaps was negligible for this purpose. It was also found that children were not educated about the utility of washing hands with soap after defecation, and it was not a common practice among them. Some children did not was their hands at all after defection.

From the stools samples pathogen were not isolated in most of the cases. It is most likely due to the fact that the cases were treated with antibiotics by private practitioners before they came to the district hospital. Though the history of such antibiotic treatment could not be elicited from majority of the patients. However infection was of mixed etiology as is evidenced by isolation of Shigella spp. as well as Vibrio Cholera from some of the samples. Ise $\mathrm{T}$ et al studied seasonal out break of diarrhoea in children in Kathmandu in 1996. Vibrio Cholera and enteropathogenic E coli were the main organisms reported.2 Similarly in a study conducted at National Kanti Children's Hospital on 1107 children suffering from diarrhoea, stool samples investigated showed $\mathrm{V}$ cholerae along with Shigella. Salmonella and pathogenic E coli.3 Cryptosporidium was identified in cases of acute diarrhoea in a study conducted by Sherchand JB et al. Like in the present study, they also found higher prevalence of cases among females. 4 Facilities were not available for Cryptosporidium isolation in our laboratory.

Almost all the hand pumps were shallow in nature and were only 10-45 feet deep. Thereby increasing chances of contamination by surface water specially during the rainy season. This is also evidenced by the laboratory analysis of the ware samples collected from the hand pumps, majority of which showed presence of coliform organisms.

Extensive electronic literature search showed that out break of GE and cholera has been occurring regularly in Nepal. But most of the reported studies have been confined to the Kathmandu Valley, which is located in the central part of the country. But no studies are carried out in the terai area. Which is the msot densely populated area of the country.

\section{CONCLUSION}

The out break of gastroenteritis in the terai region of Nepal during and after the Mansoon is a regular 
phenomenon. The contamination of drinking water source occurs commonly because of shallow nature of the hand pump. The unhygienic habits related to defecation contributes to the out break. And the health care delivery infrastructure is found wanting in controlling the out breaks as well as treating the cases. Seven deaths including one in the hospital may not be pointing to a high mortality but all GE deaths are preventable with prompt treatment. Therefore, it is pertinent that GE out breaks may be prevented by simply promoting sanitation and spreading awareness about hygienic practices related to defecation and handling of drinking water.

\section{REFERENCE}

1. A nonymous, $D$ iarrhoeal diseases. $G$ astroenteritis and cholera epidemic, 1991. W kly E pidemiol R ec S ep 11; 67(37): 273-6.

2. I se T, P okharel B M, R awal S, Shrestha $R$ S and $D$ hakhwa J R. 0 utbreaks of cholera in $K$ athmandu $\checkmark$ alley in N epal. J T ropical P ediatr 19960 ct; 42(5): 305- 7 .

3. P okhrel $B M, K$ ubo $T, O$ utbreaks of cholera in $N$ epal. Southeast A sian J T rop M ed P ublic $H$ eal th 1996 S ep; 27(3): 574-9.

4. Sherchand J B, Shrestha M P. P reval ence of $C$ ryptosporidium infection and diarrhae in $N$ epal. J D iarrhoeal D is R es 1996 J un; 14 (2): 81- 4.

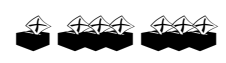

Clip from Other Journal
The radiation dose to the lens of the eye is around 30 mSv (equivalent of 1500 chest radiographs)
in CT scan of the head. Similarly, the CT scan of the chest gives a radiation dose equivalent to
400 chest X-rays (chest tomography $=8$ mSv; chest radiography = 0.02 mSv). CT scan of the
thoracic spine, mediastinum, abdomen, liver, pancreas, kidney, lumbar spine, and pelvis is
associated with effective radiation doses from more than 5 mSv (equivalent to 250 chest
radiographs) to as high as 30 mSv (equivalent to 1500 chest X-rays). The dose to the breast in
many thoracic examinations ranges from 18 mSv (900 chest radiographs) to 33 mSv (1650 chest
radiographs). Thus, the referring clinicians should remember that computed tomography (CT)
scan examinations are not without risk and should ensure that the examinations they request are
really necessary - and the most appropriate. ${ }^{1}$

JNMA, July - September, 2002, 41 